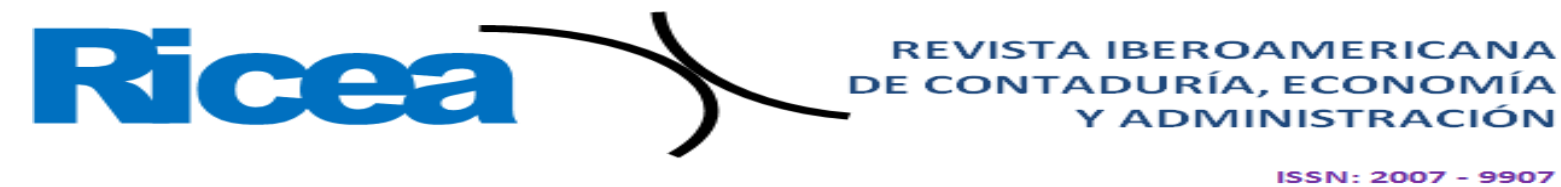

\title{
El uso de modelos de redes y modelos de transporte para la optimización y reducción de tiempos y costos de transporte en la Comercializadora Gonac S. A de C. V.
}

\begin{abstract}
The use of network models and transport models for the optimization and reduction of transport times and costs in the Comercializadora Gonac S. A de C. V.

$O$ uso de modelos de rede e modelos de transporte para a otimização e redução de tempos e custos de transporte na Comercializadora Gonac S. A de C. V.
\end{abstract}

\author{
María Elizabeth Serrano Cervantes \\ Instituto Tecnológico Superior de Misantla, México \\ eli_ser_89@hotmail.com \\ https://orcid.org/0000-0003-4309-0838 \\ Luis de Jesús Montero García \\ Instituto Tecnológico Superior de Perote, México \\ jesusmonterogarcia@hotmail.com \\ https://orcid.org/0000-0001-5723-0024
}

\section{Resumen}

El objetivo del presente artículo es enseñar los resultados en torno a cómo en la Comercializadora Gonac S. A de C. V. se pueden recortar los tiempos y costos de traslado de sus productos a través del uso de técnicas de optimización de redes y modelos de transporte denominados ruta más corta, modelo de transporte y árbol de expansión mínima. Cada una de estas técnicas fue resuelta de manera matemática y por medio del software Win QSB, lo que facilitó el proceso de programación y asignación de rutas en el área de logística de la mencionada empresa. Junto con la aplicación de estas técnicas se emplearon fórmulas para calcular el consumo de combustible de cada unidad de la dicha compañía. Estas técnicas de optimización de redes y modelos de transporte permitieron visualizar las 


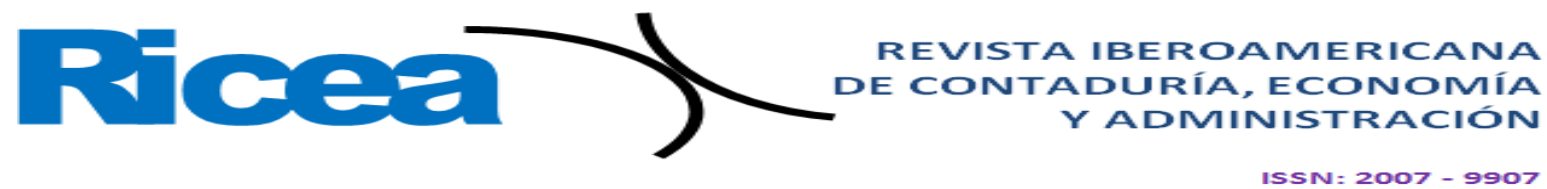

ventajas y desventajas que se tienen al programar las diferentes rutas. En tal sentido, se debe prever que cada una de estas es diferente, por lo que las condiciones para programar y asignar las rutas de transporte varían, ya que se deben tomar en cuenta la cantidad y el volumen del producto solicitado por el cliente, así como el tiempo y la distancia recorrida, pues de esa manera se podrá escoger la unidad que debería trasladar determinado pedido a su destino, con lo cual se podrían disminuir los costos.

Palabras clave: árbol de expansión mínima, investigación de operaciones, logística y cadena de suministros, modelo de transporte, modelos de redes, ruta más corta, Win QSB.

\section{Abstract}

The objective of this article is to show the results about how the Gonac S. A de CV sales company can reduce the time and costs of moving its products through the use of network optimization techniques and transport models called route shorter, transport model and minimum expansion tree. Each of these techniques was solved mathematically and through the Win QSB software, which facilitated the process of programming and assigning routes in the logistics area of the aforementioned company. Along with the application of these techniques formulas were used to calculate the fuel consumption of each unit of the said company. These techniques of optimization of networks and transport models allowed to visualize the advantages and disadvantages that are had when programming the different routes. In this regard, it must be anticipated that each of these is different, so the conditions for programming and assigning transport routes vary, since the quantity and volume of the product requested by the customer must be taken into account, as well as the time and distance traveled, because that way you can choose the unit that should move a certain order to its destination, which could reduce costs.

Keywords: minimum expansion tree, operations research, logistics and supply chain, transport model, network models, shorter route, Win QSB.

Keywords: network models, shortest route, transport model and minimum expansion tree, logistics and supply chain, operations research, Win QSB. 


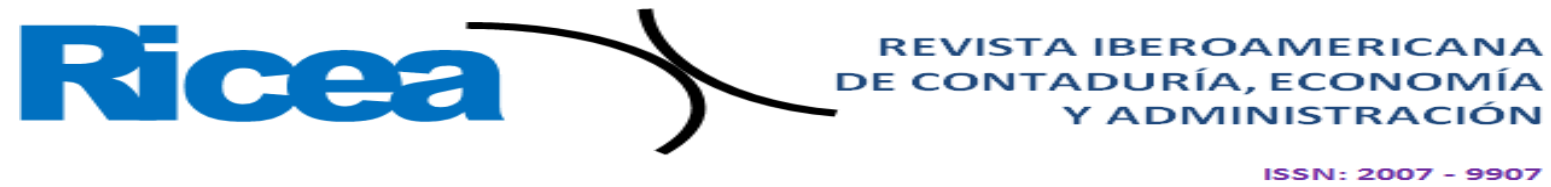

\section{Resumo}

O objetivo deste artigo é mostrar os resultados de como a empresa de vendas Gonac $\mathrm{S}$. A de CV pode reduzir o tempo e os custos de movimentação de seus produtos através do uso de técnicas de otimização de rede e modelos de transporte chamados de rota. menor, modelo de transporte e árvore de expansão mínima. Cada uma destas técnicas foi resolvida matematicamente e através do software Win QSB, o que facilitou o processo de programação e atribuição de rotas na área de logística da empresa supracitada. Juntamente com a aplicação dessas técnicas, foram utilizadas fórmulas para calcular o consumo de combustível de cada unidade da referida empresa. Estas técnicas de otimização de redes e modelos de transporte permitiram visualizar as vantagens e desvantagens que se tem ao programar as diferentes rotas. A este respeito, deve-se antecipar que cada um deles é diferente, de modo que as condições de programação e atribuição de rotas de transporte variam, uma vez que a quantidade e o volume do produto solicitado pelo cliente devem ser levados em consideração, assim como como o tempo e a distância percorrida, porque dessa forma você pode escolher a unidade que deve mover uma determinada ordem para o seu destino, o que poderia reduzir os custos.

Palavras-chave: árvore de expansão mínima, pesquisa operacional, logística e cadeia de suprimentos, modelo de transporte, modelos de rede, rota mais curta, Win QSB.

Fecha Recepción: Junio $2018 \quad$ Fecha Aceptación: Octubre 2018

\section{Introducción}

En 2015, las autoridades mexicanas firmaron en París un acuerdo cuyo objetivo central se enfocó en que $35 \%$ de la energía consumida para el año 2025 deberá provenir de fuentes renovables, pues de esta manera se podrá reducir la emisión de gases de efecto invernadero, el cual es producido

principalmente por el sector del trasporte. Para conseguir esta meta, por supuesto, México deberá trabajar en la investigación de otras fuentes de energía, como las provenientes de biocombustibles como la higuerilla, el maíz, la caña de azúcar, el trigo, el sorgo y los cítricos, los cuales no solo tienen un menor impacto ambiental, sino que también pueden servir para reducir los costos invertidos en 


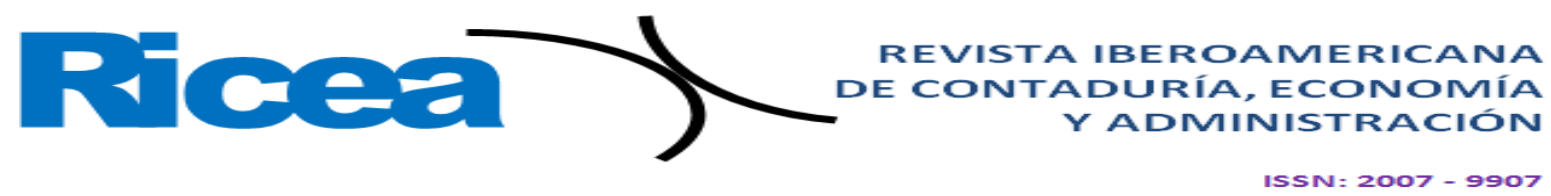

combustibles fósiles para distribuir los productos a nivel local, nacional e internacional. Boluda Aguilar, M. y López Gómez, A. (2013).

En el país, sin embargo, hasta el momento solo existe una empresa certificada (llamada Fuel Flex) en la comercialización e investigación de nuevos biocombustibles, mientras que a nivel institucional y gubernamental el Instituto Politécnico Nacional cuenta con el Laboratorio Nacional de Desarrollo y Aseguramiento de la Calidad de Biocombustibles (Landacbio), el cual ofrece servicios analíticos y de consultoría especializada, investigación científica, desarrollo tecnológico y capacitación de recursos humanos para maximizar la productividad y competitividad de la industria nacional bajo los principios de sustentabilidad. Asimismo, y por iniciativa de la Secretaría de Energía (Sener) y el Consejo Nacional de Ciencia y Tecnología (Conacyt) ha surgido el Centro Mexicano de Innovación en Bioenergía (Cemie-Bio), integrado por cinco clústeres dedicados a la investigación, desarrollo e innovación en distintos tipos de biocombustible. Gay y García, C. (2014).

Lo anterior significa que aún falta invertir mucho para desarrollar fuentes alternas de energía. Mientras tanto, el uso de combustibles fósiles seguirá siendo uno de los principales ejes para dinamizar la economía del país, de ahí que se deban presentar estrategias para disminuir el impacto ambiental y logístico que implica trasladar los productos que requiere la sociedad.

\section{El problema}

La empresa Comercializadora Gonac S. A de C. V. es una compañía moderna mexicana que procura la mejora continua de sus servicios, lo cual se evidencia en el empleo de tecnologías y técnicas de ingeniería para simplificar la operatividad de sus actividades, que consisten en la fabricación de botanas y bebidas que son comercializadas en México, Estados Unidos y Centroamérica. Esta tiene dos plantas: una en la ciudad de Huamantla, Tlaxcala, C. P. 90500, específicamente en la calle Feria Nacional, número 7, Cd. Industrial Xicohtencatl II, y otra planta en la ciudad de Monterrey, ubicada en la calle Brasil, número 205, en el Parque Industrial Martel Santa Catarina, Nuevo León C. P.66358.

La compañía actualmente se encuentra trabajando con seis diferentes marcas de productos de botanas y bebidas (Kiubo, King Citrus, Chechitos, Chechi Fresco, Fruti King y Ecolive) y con más de cien productos en el mercado. Para garantizar los estándares de calidad, Gonac se basa en el esquema 


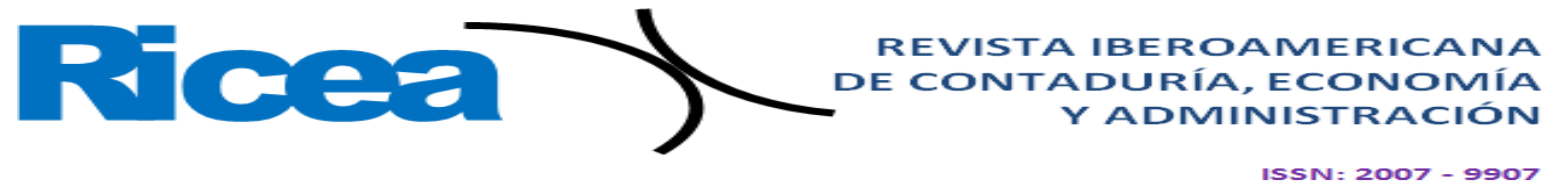

de calidad y mejora continua, según se establece en la norma NOM-251-SSA1-2009, la cual determina las prácticas de higiene para el proceso de alimentos, bebidas o suplementos alimenticios.

El departamento de logística de la empresa Gonac se encarga de desarrollar, mediante un programa de embarques, aquellas actividades que están vinculadas con la asignación de rutas y envíos de productos a los destinos requeridos. En este proceso se van monitoreando y rastreando las unidades mediante un sistema satelital GPS llamado Qtracs Omnitraqs. De esta manera la empresa procura que sus mercancías lleguen a los clientes de forma correcta y en lapso establecido.

Pese a este control, sin embargo, se ha detectado un problema relacionado con el retardo en la entrega de los pedidos de los clientes, así como en los costos que implica el traslado de la mercancía hacia sus destinos finales (figura 1).

Figura 1. Diagrama de causa y efecto

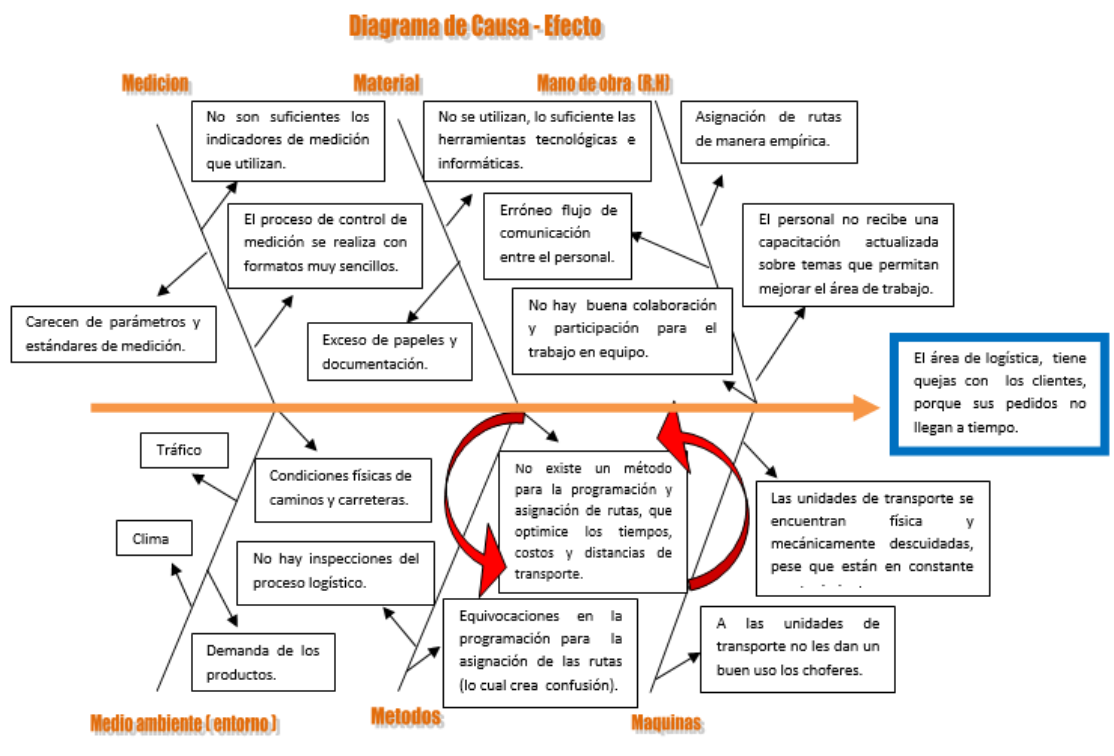

Fuente: Elaboración propia

Por este motivo, el objetivo del presente artículo es enseñar los resultados en torno a cómo se pueden recortar los tiempos y costos de traslado de productos a través del uso de técnicas de optimización de redes y modelos de transporte denominados ruta más corta, modelo de transporte y árbol de expansión mínima. 


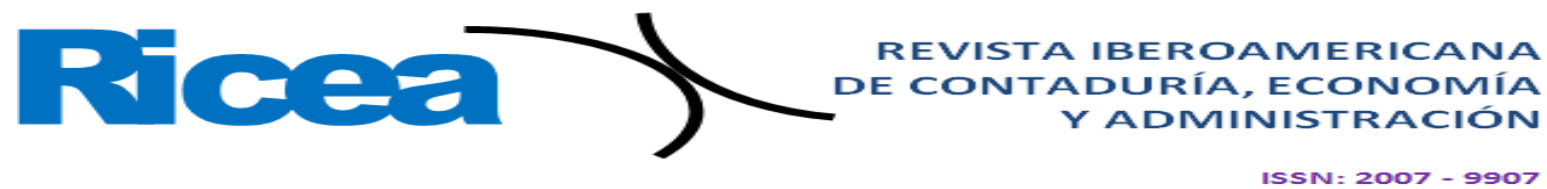

\section{Método}

Cada una de las anteriores técnicas fue resuelta de manera matemática y por medio del software Win QSB, lo cual facilitó el proceso de programación y asignación de rutas en el área de logística en la empresa Comercializadora Gonac S. A de C. V.

Junto con la aplicación de estas técnicas se emplearon fórmulas para calcular el consumo de combustible de cada unidad de la mencionada empresa. Además, se consideraron las tarifas de peaje o casetas de cobro que la Secretaría de Comunicaciones y Transportes establece en México, específicamente las del lapso 2013-2014.

Ahora bien, para poder dar solución a la problemática en la empresa y optimizar los costos y tiempos de transporte se utilizaron las siguientes técnicas de la ingeniería industrial enfocadas en la investigación de operaciones, logística y cadena de suministros.

\section{Logística}

Son tareas necesarias para planificar, implementar y controlar el flujo físico de materiales, productos terminados e información relacionada desde los puntos de origen hasta los de consumo para satisfacer las necesidades del cliente de manera rentable. En palabras de Ballou (2004), "es el proceso de administrar estratégicamente el flujo y almacenamiento eficiente de las materias primas, de las existencias en el proceso y de los bienes terminados del punto de origen al de consumo” (p. 7).

\section{Investigación de operaciones}

Según Prawda (2004), "la investigación de operaciones es una aplicación de la metodología científica que a través de modelos matemáticos busca representar de forma real un problema y dar solución al mismo" (p. 936), lo cual permite hacer un análisis detallado para la toma de decisiones en cualquier organización u empresa.

\section{Modelos de redes}

Un modelo es una representación o abstracción de una situación u objeto real que muestra las relaciones (directas o indirectas), así como las interrelaciones de la acción y la reacción en términos de causa y efecto. Al respecto, Taha (2004) considera que "la modelación de redes permite la 


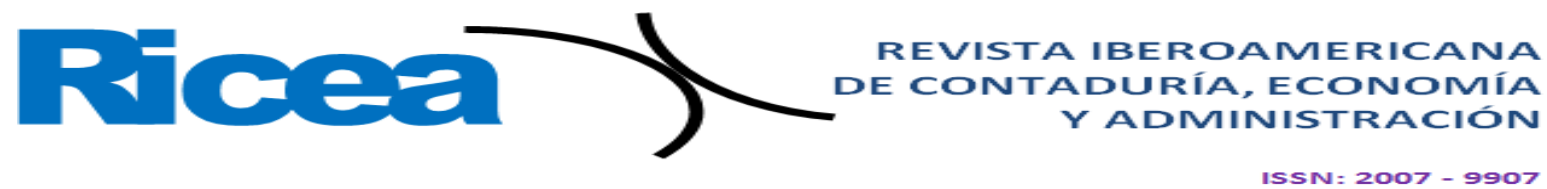

resolución de múltiples problemas de programación matemática mediante la implementación de algoritmos especiales creados para tal fin, conocidos como algoritmos de optimización de redes" (p. 213). Algunos de los problemas resueltos de manera más habitual mediante la modelación de redes se vinculan con el modelo de transporte, la ruta más corta, el modelo de costo mínimo, entre otros. Una red es la unión de dos o más puntos llamados nodos, los cuales se encuentran ligados por líneas denominadas arcos.

\section{Optimización de redes}

Este concepto busca a través de modelos matemáticos determinísticos disminuir el tiempo y la distancia entre dos o más puntos. Este surge con el propósito de optimizar las redes de transporte y la energía eléctrica para el suministro de combustible.

\section{Optimización de rutas}

Sobre este concepto, Castellanos Ramírez (2009) explica lo siguiente:

Podría entenderse por optimización de rutas todas aquellas acciones que contribuyan a la mejora de la función de distribución, bien sea en términos de nivel de servicio, mejora de la calidad, reducción de costes. La optimización de rutas es un concepto que toca necesariamente los tres niveles de decisión, si bien, habitualmente adquiere mayor importancia en las decisiones de carácter más táctico y operacional, esto es, en el momento de optimizar modelos ya existentes o adaptarlos ante la necesidad de incorporar nuevos productos o clientes en los flujos de distribución ya implantados (p. 260).

\section{La ruta más corta}

El objetivo de este método es simplificar y acortar distancias para reducir los tiempos y los costos (Taha, 2004). A través de este se representa de forma real el problema, así como su solución de manera gráfica, lo cual permite ampliar el panorama para tomar decisiones en cuanto al reparto de 


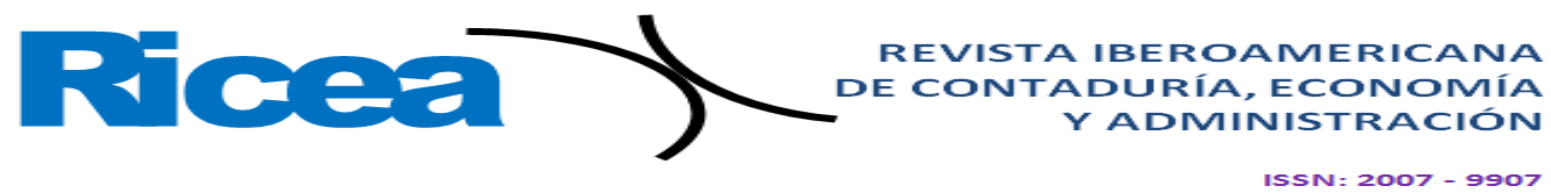

los productos de la empresa. La ruta más corta, en otras palabras, procura minimizar la distancia recorrida, así como el costo y el tiempo invertido en una secuencia de actividades.

\section{Método del árbol de extensión mínima}

Para Hillier (2006) "el método del árbol de extensión o expansión mínima consiste en encontrar las conexiones más eficientes o más cortas entre todos los nodos de una red” (p. 318). Es decir, conecta todos los puntos de una red de manera que la distancia y el tiempo disminuyan, lo cual recorta los costos. En este modelo de optimización de redes no existen ciclos. El árbol de expansión mínima, por ende, es un modelo que consiste en enlazar todos los nodos de la red de forma directa o indirecta para que la longitud total de los arcos o ramales sea mínima.

\section{Modelo de transporte}

El modelo de transporte busca determinar un plan de transporte de una mercancía de varias fuentes a varios destinos. Los datos del modelo son los siguientes:

1. Nivel de oferta en cada fuente y la cantidad de demanda en cada destino.

2. El costo de transporte unitario de la mercancía a cada destino.

Como solo hay una mercancía, un destino puede recibir su demanda de una o más fuentes. El objetivo del modelo es determinar la cantidad que se enviará de cada fuente a cada destino para disminuir el costo del transporte total (Castellanos Ramírez, 2009).

Este modelo parte de la premisa de que el costo del transporte en una ruta es directamente proporcional al número de unidades transportadas. En este sentido, la definición de unidad de transporte varía dependiendo de la mercancía trasladada, de ahí que haya $m$ fuentes y $n$ destinos, los cuales son representados por un nodo. Los arcos constituyen las rutas que enlazan las fuentes y los destinos. El arco $(i, j)$ que une a la fuente $i$ con el destino $j$ contiene dos clases de información: el costo de transporte $c i j$ por unidad y la cantidad transportada $x i j$. La cantidad de oferta en la fuente $i$ es $a i$ y la cantidad de demanda en el destino $j$ es $b j$. El objetivo del modelo es determinar las incógnitas $x i j$ 


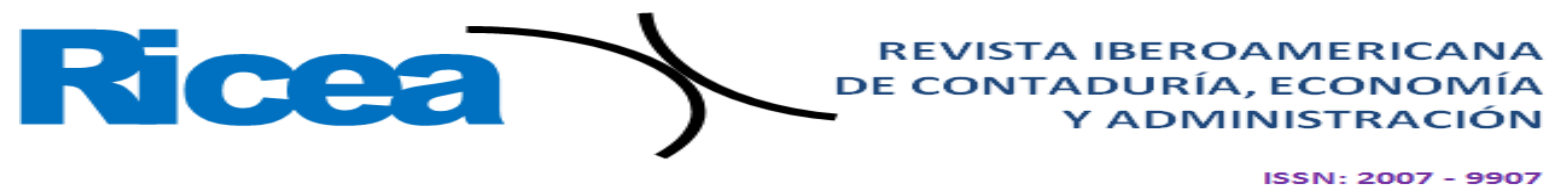

para reducir el costo total de transporte y satisfacer, al mismo tiempo, las restricciones de oferta y demanda.

\section{Programa Win QSB}

WinQSB es un sistema interactivo que ayuda a la toma de decisiones. Este contiene herramientas muy útiles para resolver distintos tipos de problemas en el campo de la investigación operativa. El sistema está formado por múltiples módulos (uno para cada tipo de modelo o problema). Es un software que permite definir los problemas de planificación en tres grupos: modelo simple, modelo de transporte y modelos de programación lineal, por lo que es útil para especificar la disponibilidad de horas extras, demoras en la entrega de pedidos, subcontratación, pérdida de ventas, así como contratos y despidos de recursos.

Explicado lo anterior, a continuación se presentan las fórmulas usadas para calcular el consumo de combustible de cada una de las rutas asignadas por la empresa, para lo cual se consideró el rendimiento de cada tipo de unidad de transporte, los kilómetros de distancia entre cada destino y el costo unitario del combustible:

\section{Ecuación 1}

$$
\text { consumo de combustible en litros }=\frac{\mathrm{km}}{\text { rendimiento de la unidad }}
$$

\section{Ecuación 2}

costos de consumo de combustible $=\frac{\mathrm{km}}{\text { rendimiento de la unidad }}($ costo unitario del combustible $)$

En la tabla1 se muestran los rendimientos de las unidades de transporte establecidos y calculados por la empresa, para lo cual se tomó en cuenta si el vehículo necesitaba ajuste o tenía algún problema con el consumo excesivo de combustible. 


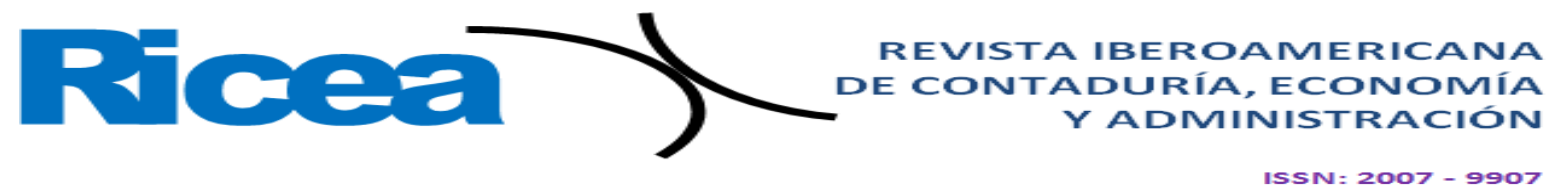

Tabla 1. Rendimientos de las unidades

\begin{tabular}{|c|c|}
\hline Tipo de unidad & Rendimiento \\
\hline Camioneta & 5.8 \\
\hline Torton & 3.3 \\
\hline Rabón & 4.2 \\
\hline Tráiler & 2.7 \\
\hline Full & 2.2 \\
\hline Palmex & 10 \\
\hline
\end{tabular}

Fuente: Comercializadora Gonac (2012)

Para obtener el rendimiento de las unidades se empleó la siguiente fórmula:

Ecuación 3

$$
\frac{k m \text { recorridos } B-k m \text { recorridos } A}{\text { litros } B}=\text { rendimiento }
$$

Es decir, el rendimiento del vehículo es igual a los kilómetros recorridos la última vez por la unidad menos los kilómetros recorridos anteriormente, y este resultado luego es dividido entre los litros de combustible que cargó la última vez la unidad.

Ahora bien, la importancia de aplicar los modelos de redes y los modelos de transportes en la programación de rutas para distribuir productos de la empresa Gonac radica en que se intenta no solo disminuir los costos que implica el uso diario de una flotilla conformada por más de 200 unidades, sino también reducir el tiempo invertido en la entrega de sus productos o servicios. Para ello, este tipo de modelos se basa en algoritmos matemáticos que ayudan a tomar decisiones objetivas en cuanto a logística y cadena de suministro, todo ello expresado en números que reflejan costo, tiempos y distancias.

Sin embargo, también vale destacar que dicho modelo no considera algunos factores, como son el tráfico, el clima, las condiciones de caminos y carreteras, el funcionamiento de las unidades, el volumen y la capacidad de carga de cada camión, las ventas y pronósticos de ventas, la generación de pedidos, el tiempo en que las unidades están inactivas, las tarifas de casetas de peaje, los indicadores de medición de transporte, el monitoreo y rastreo de las unidades, así como la variabilidad de los 


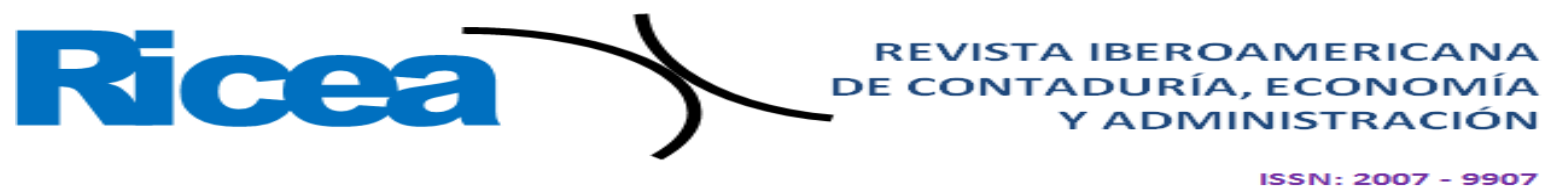

diferentes destinos para la creación de rutas, las fechas de entrega del producto, los accidentes viales, el tiempo de carga y la descarga del producto, o la zona geográfica y económica del país.

\section{Resultados}

\section{Aplicación del modelo de la ruta más corta}

Para este modelo se consideró la ruta Huamantla-Monterrey, de modo que la siguiente red muestra las conexiones y kilómetros que existen entre un destino y otro (figura 2):

Figura 2. Red de la ruta Huamantla-Monterrey

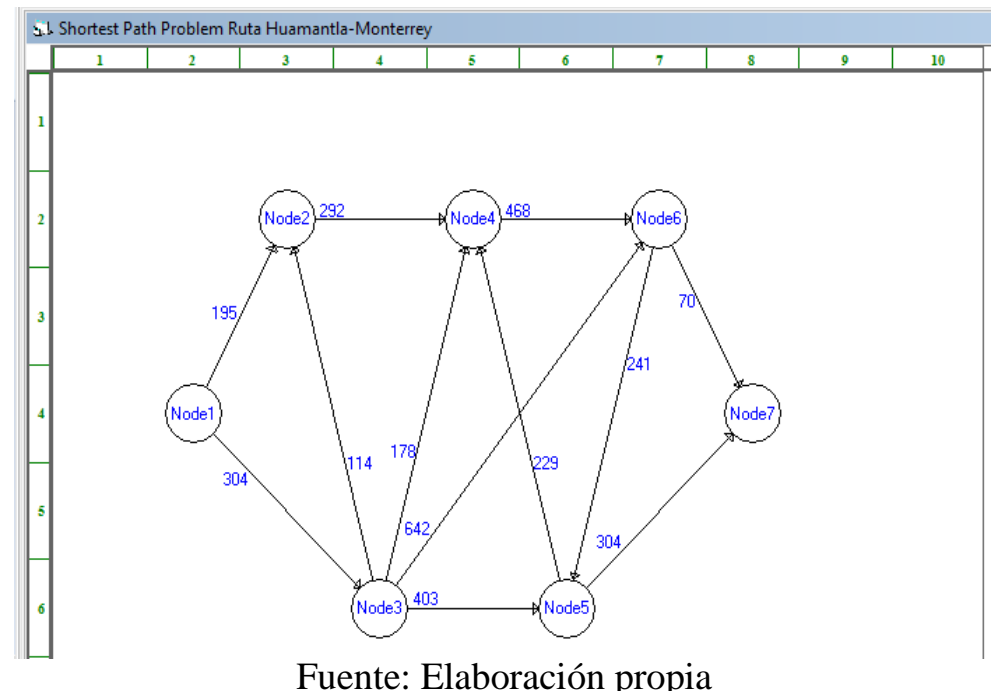

La siguiente red (figura 3) representa el recorrido que debería de realizar la unidad de transporte asignada en la ruta Huamantla-Monterrey, la cual es establecida por la empresa y considerada como base para fijar dicho método. 


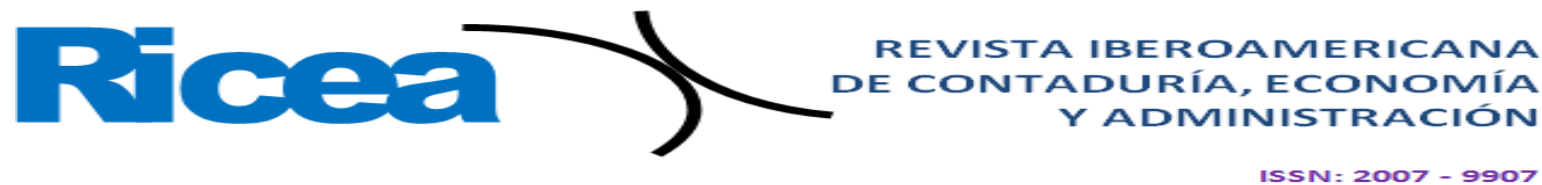

Figura 3. Red de la ruta Huamantla-Monterrey asignada por la empresa

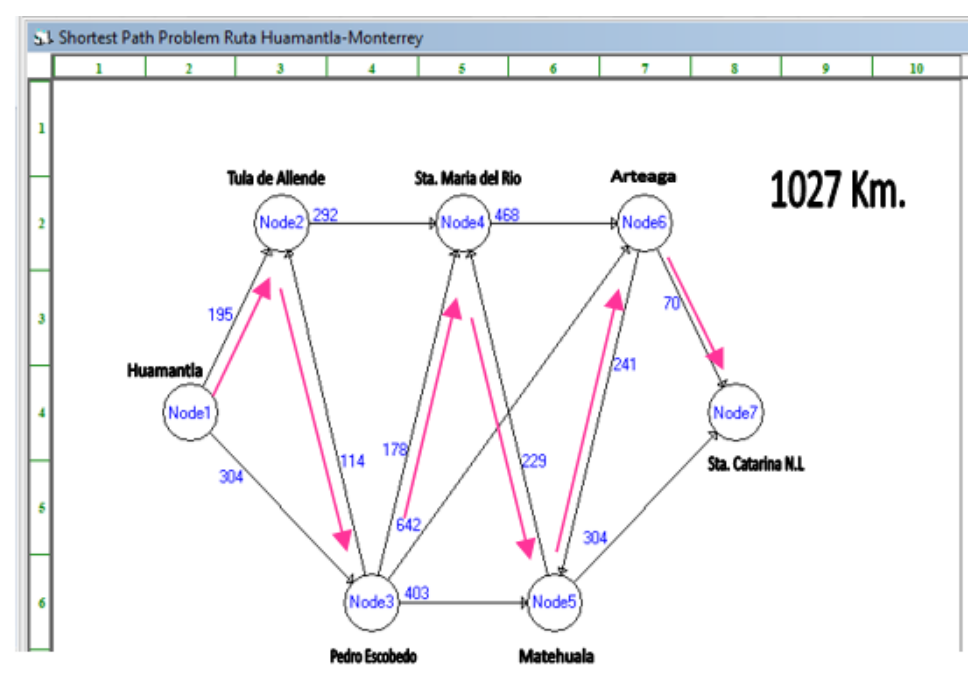

Fuente: Elaboración propia

En la tabla 2 se enseñan los destinos o poblaciones por donde se debe pasar para llegar al destino final (planta de Monterrey), así como las distancias existentes entre un destino y otro para poder definir a través de la red la ruta más viable.

Tabla 2. Muestra los destinos y kilometrajes de la ruta Huamantla-Monterrey

\begin{tabular}{|c|c|c|}
\hline Desde & Hacia & km \\
\hline Huamantla & Tula de Allende & $195 \mathrm{~km}$ \\
\hline Tula de Allende & Pedro Escobedo & $114 \mathrm{~km}$ \\
\hline Pedro Escobedo & Santa María del Rio & $178 \mathrm{~km}$ \\
\hline Santa María del Río & Matehuala & $229 \mathrm{~km}$ \\
\hline Matehuala & Arteaga & $241 \mathrm{~km}$ \\
\hline Arteaga & Santa Catarina NL. & $70 \mathrm{~km}$ \\
\hline \multicolumn{2}{|r|}{ Total } & $1027 \mathrm{~km}$ \\
\hline
\end{tabular}

Fuente: Comercializadora Gonac (2012) 


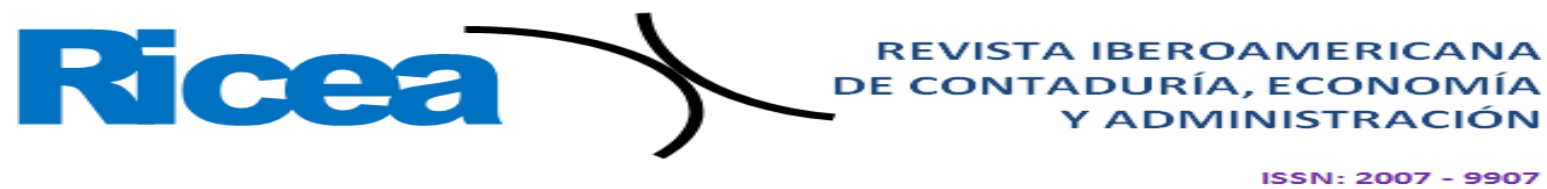

Figura 4. Mapa de la Ruta Huamantla-Monterrey

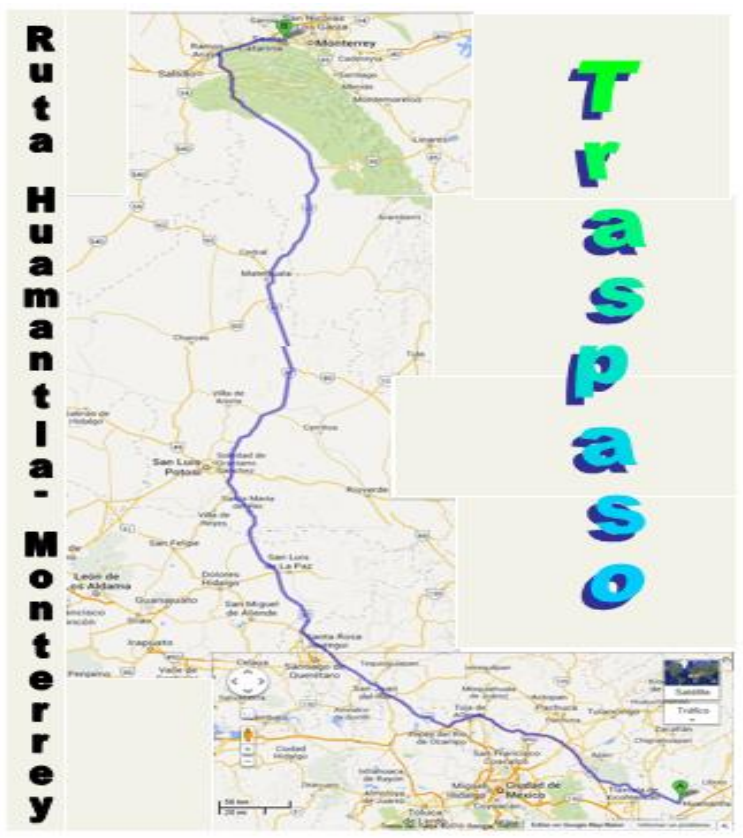

Fuente: Elaboración propia

La siguiente red (figura 5) muestra el recorrido en kilómetros, así como la optimización de las distancias entre Huamantla y Monterrey a través del método de la ruta más corta.

Figura 5. Red propuesta por el método la ruta más corta

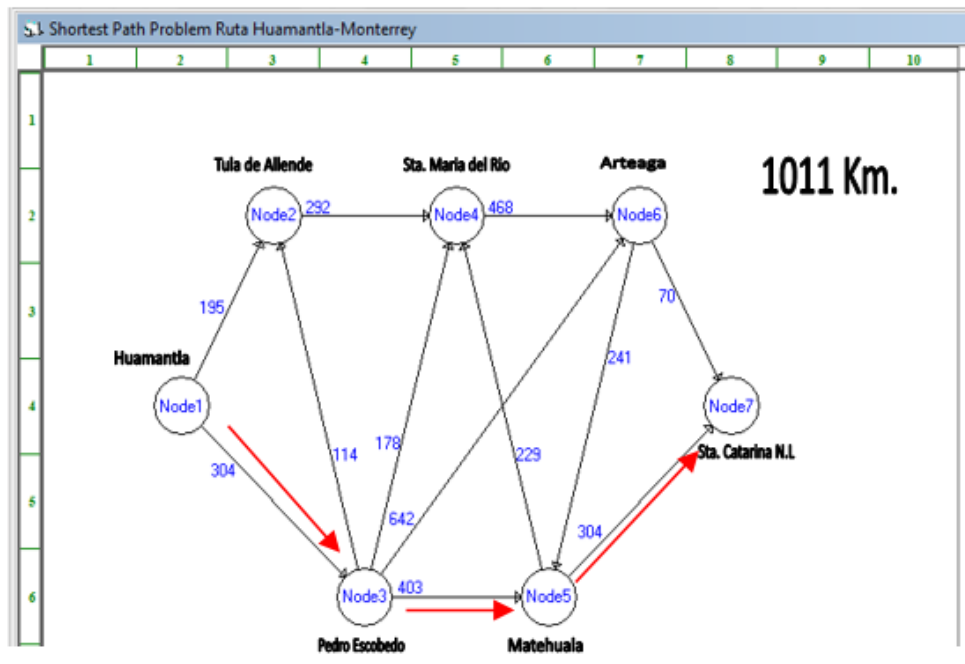

Fuente: Elaboración propia 


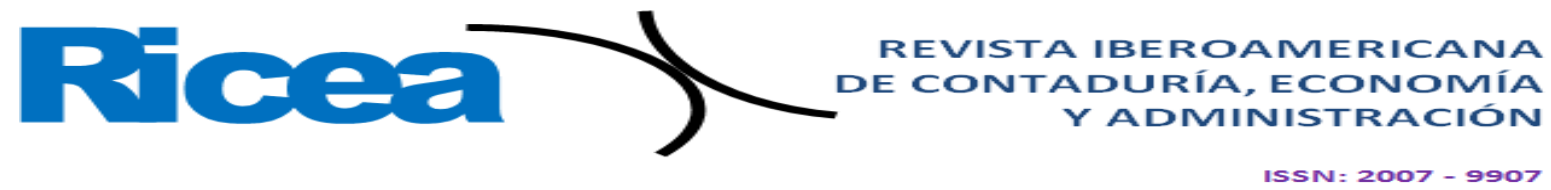

A continuación (figura 6), y con ayuda del programa Win QSB, se presenta la resolución del algoritmo de la ruta Huamantla-Monterrey, mediante el cual se consigue un resultado igual al anterior.

Figura 6. Solución del algoritmo de la ruta más corta entre Huamantla-Monterrey con el Win QSB

\begin{tabular}{|c|c|c|c|c|c|c|c|c|}
\hline \multicolumn{9}{|c|}{ 21 Shortest Path Problem RUTA DE HUAMANTLA-MONTERREY } \\
\hline Node7 : Node5 & $x$ P 304 & & & & & & & \\
\hline & From $\backslash$ To & Node1 & Node2 & Node3 & Node4 & Node5 & Node6 & Node7 \\
\hline & Node1 & & 195 & 304 & & & & \\
\hline & Node2 & 195 & & 114 & 292 & & & \\
\hline & Node3 & 304 & 114 & & 178 & 403 & 642 & \\
\hline & Node4 & & 292 & 178 & & 229 & & \\
\hline & Node5 & & & 403 & 229 & & 241 & 304 \\
\hline & Node6 & & & 642 & 468 & 241 & & 70 \\
\hline & Node7 & & & & & 304 & 70 & \\
\hline
\end{tabular}

\begin{tabular}{|c|c|c|c|c|}
\hline \multicolumn{5}{|l|}{ 31 Solution for Shortest Path Probl } \\
\hline $1 /$ & Node1 & Node3 & 304 & 304 \\
\hline 2 & Node3 & Node5 & 403 & 707 \\
\hline 3 & Node5 & Node7 & 304 & 1011 \\
\hline & Fiom NodeT- & Io Node7 & $=$ & 1011 \\
\hline & From Node1 & To Node2 & - & 195 \\
\hline & From Node1 & To Node3 & $=$ & 304 \\
\hline & From Node1 & To Node4 & $=$ & 482 \\
\hline & From Node1 & To Node5 & - & 707 \\
\hline & From Node1 & To Node6 & $=$ & 946 \\
\hline
\end{tabular}

Fuente: Elaboración propia

\section{Resultado $7 \longrightarrow 5 \longrightarrow 3 \longrightarrow 1$}

Ruta: Huamantla, Pedro Escobedo, Matehuala, Santa Catarina NL.

$$
\text { Distancia } 304+403+304=1011 \mathrm{~km}
$$

Kilómetros reducidos: 1027 km -1011 km = 16 km

En la tabla 3 aparece resuelto el algoritmo de la ruta más corta, el cual arrojó los siguientes resultados: 


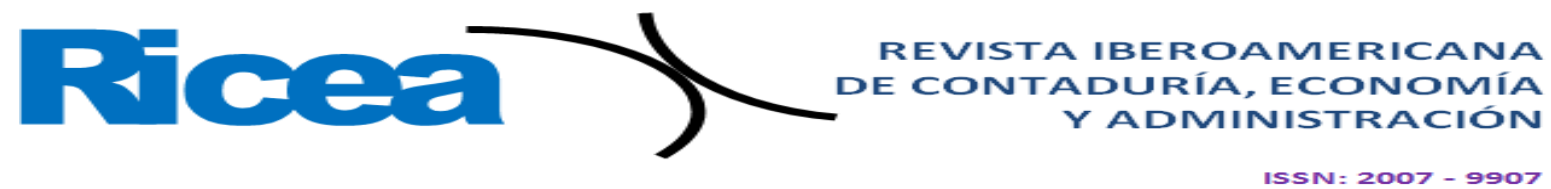

Tabla 3. Algoritmo de la ruta más corta entre Huamantla-Monterrey

\begin{tabular}{|c|c|c|}
\hline Nodo & $u_{i j}$ & Etiqueta \\
\hline $\begin{array}{c}1 \\
\text { Huamantla } \\
\end{array}$ & $u_{1} \min =(0+0)=0$ & {$[0,0]$} \\
\hline $\begin{array}{c}2 \\
\text { Tula de Allende }\end{array}$ & $u_{21} \min =(0+195)=195$ & {$[195,1]$} \\
\hline \multirow[t]{2}{*}{$\begin{array}{c}3 \\
\text { Pedro Escobedo } \\
\end{array}$} & $u_{31} \min =(0+304)=304$ & {$[304,1]$} \\
\hline & $u_{32} \min =(195+114)=309$ & \\
\hline \multirow[t]{2}{*}{$\begin{array}{c}4 \\
\text { Santa María del Río }\end{array}$} & $u_{42} \min =(195+292)=487$ & \\
\hline & $u_{43} \min =(304+178)=482$ & {$[482,3]$} \\
\hline \multirow[t]{2}{*}{$\begin{array}{c}5 \\
\text { Matehuala }\end{array}$} & $u_{53} \min =(304+403)=707$ & {$[707,3]$} \\
\hline & $u_{54} \min =(482+229)=711$ & \\
\hline \multirow[t]{3}{*}{$\begin{array}{c}6 \\
\text { Arteaga } \\
\end{array}$} & $u_{63} \min =(304+642)=946$ & {$[946,3]$} \\
\hline & $u_{64} \min =(482+468)=950$ & \\
\hline & $u_{65} \min =(707+241)=948$ & \\
\hline \multirow[t]{2}{*}{$\begin{array}{c}7 \\
\text { Santa Catarina NL. }\end{array}$} & $u_{75} \min =(707+304)=1011$ & {$[1011,5]$} \\
\hline & $u_{76} \min =(946+70)=1016$ & \\
\hline
\end{tabular}

Fuente: Elaboración propia

Resultado: $16 \mathrm{~km}$ reducidos

Considerando que se envió un tráiler para recorrer esta ruta con un rendimiento de 2.7 y con un costo unitario de diésel de \$12.70, se calculó el consumo de combustible comparando la ruta asignada por la empresa y el método aplicado, como se muestra en las siguientes ecuaciones:

Cálculo del consumo de combustible de la ruta asignada por la empresa:

consumo de combustible en litros $=\frac{1027}{2.7}=380.37$ litros

costos de consumo de combustible $=\frac{1027}{2.7}(12.70)=4830.69$ 


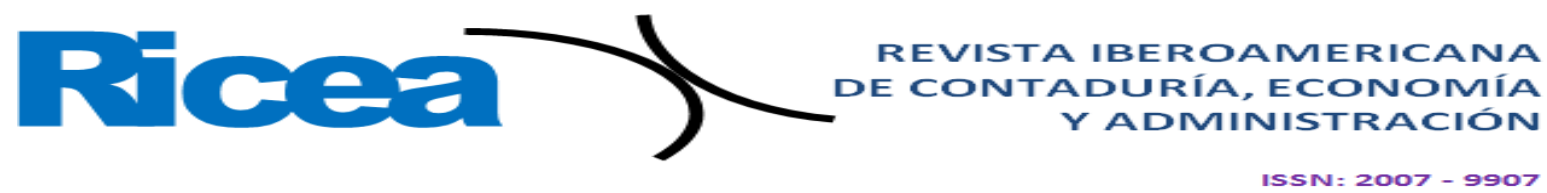

Cálculo del consumo de combustible de la ruta obtenida por el método de la ruta más corta:

consumo de combustible en litros $=\frac{1011}{2.7}=374.44$ litros

costos de consumo de combustible $=\frac{1011}{2.7}(12.70)=4755.38$

A continuación, se presenta la cantidad y el costo del consumo de combustible, los cuales se redujeron empleando el método de la ruta más corta.

$$
380.37-374.44=5.93 \text { litros } 4830.69-4755.38=75.31
$$

Tomando en cuenta el trayecto realizado por la unidad a la planta en la ida y el regreso (Huamantla-Monterrey), el consumo de combustible que se redujo fue el siguiente:

Resultado $75.31(2)=\$ 150.62$

\section{Árbol de expansión mínima}

Para optimizar las distancias de todos los nodos conectados y obtener una ruta viable para satisfacer la demanda en todos los puntos o destinos, se aplicó el método de árbol de expansión mínima en la ruta Huamantla-Tamanzunchale (figura 7): 


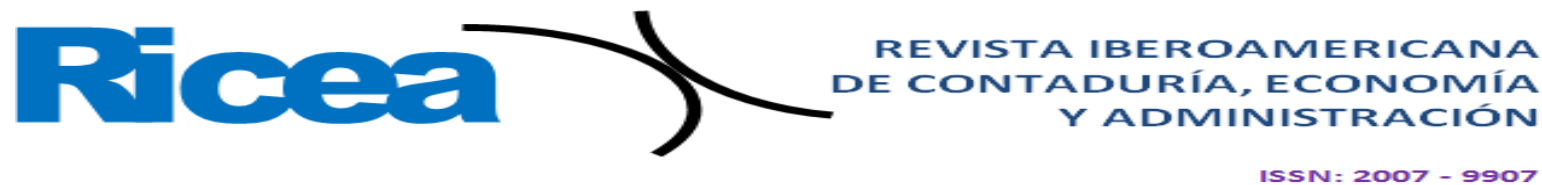

Figura 7. Red de la ruta Huamantla-Tamanzunchale

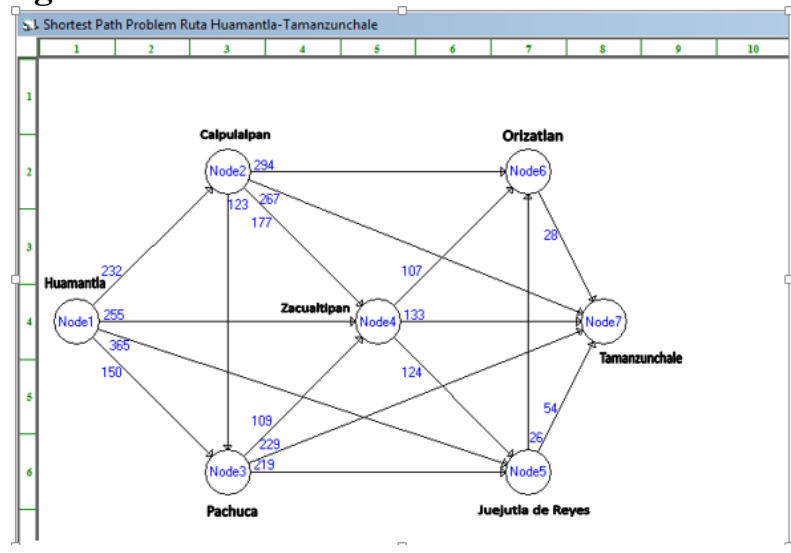

Fuente: Elaboración propia

Figura 8. Red de la ruta Huamantla-Tamanzunchale asignada por la empresa

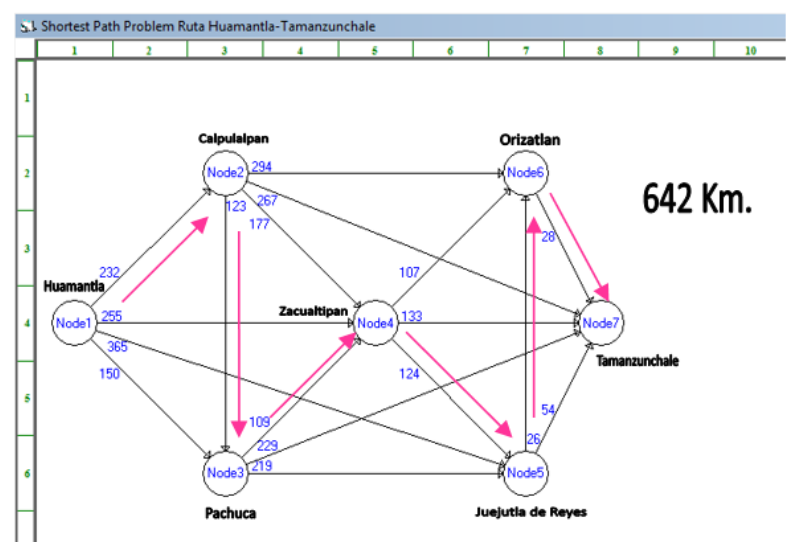

Fuente: Elaboración propia 


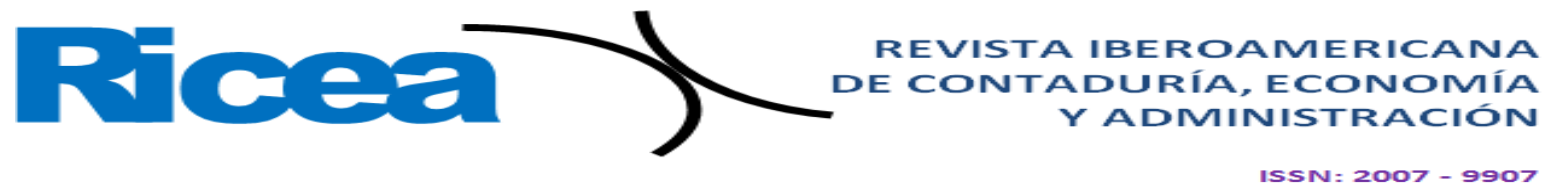

Figura 9. Mapa de la ruta Huamantla-Tamanzunchale

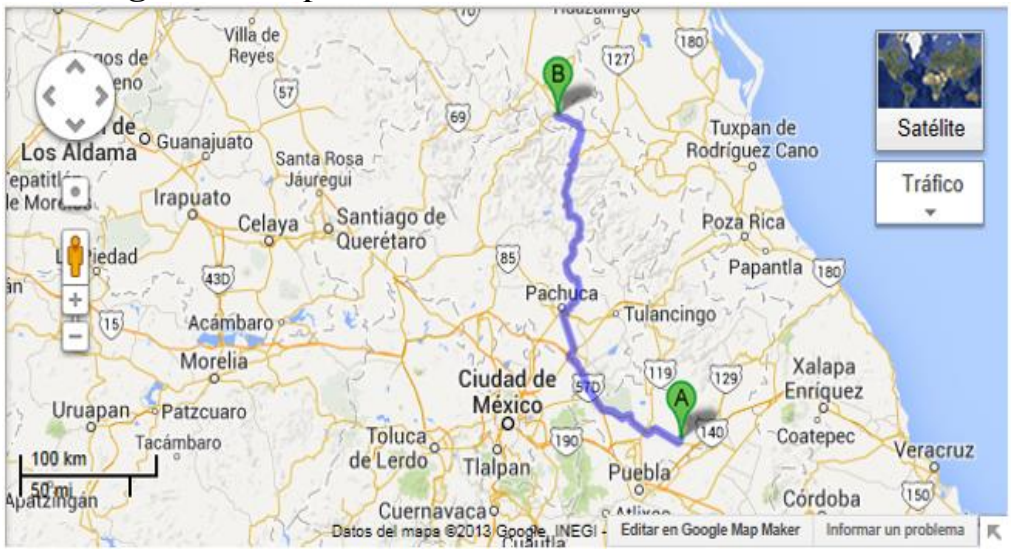

Fuente: Elaboración propia

Tabla 4. Destinos y kilometrajes de la ruta Huamantla-Tamanzunchale

\begin{tabular}{|c|c|c|}
\hline Desde & Hacia & $\mathbf{k m}$ \\
\hline Huamantla & Calpulalpan & $232 \mathrm{~km}$ \\
\hline Calpulalpan & Pachuca & $123 \mathrm{~km}$ \\
\hline Pachuca & Zacualtipan & $109 \mathrm{~km}$ \\
\hline Zacualtipan & Juejutla de Reyes & $124 \mathrm{~km}$ \\
\hline Juejutla de Reyes & Orizatlan & $26 \mathrm{~km}$ \\
\hline Orizatlan & Tamanzunchale & $28 \mathrm{~km}$ \\
\hline \multicolumn{2}{|c|}{ Total } & $642 \mathrm{~km}$ \\
\hline
\end{tabular}

Fuente: Comercializadora Gonac (2012)

La siguiente red (figura 10) presenta el recorrido y la ruta más viable de bajo costo que se obtuvo al aplicar el método de árbol de expansión mínima: 


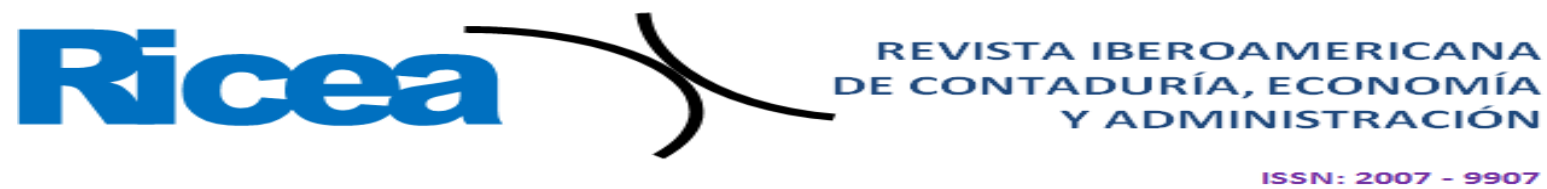

Figura 10. Red propuesta por el método de árbol de expansión mínima

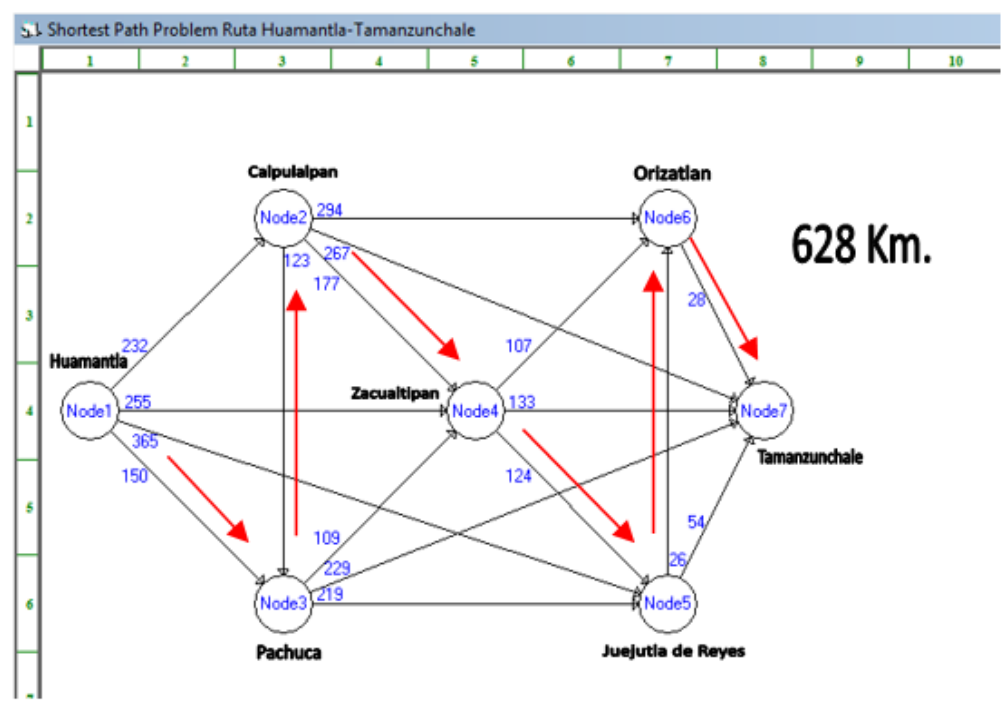

Fuente: Elaboración propia

El método del árbol de expansión mínima se resolvió con base en la tabla 5, la cual contiene los nodos y las distancias mínimas que conectan a todos los nodos de la red.

Tabla 5. Solución de la ruta Huamantla Tamanzunchale por el método de árbol de expansión mínima

\begin{tabular}{|c|c|c|}
\hline$C_{K}$ & $\overrightarrow{C_{K}}$ & Distancia \\
\hline 7 & $6,5,4,3,2,1$ & - \\
\hline 7,6 & $5,4,3,2,1$ & 28 \\
\hline $7,6,5$ & $4,3,2,1$ & 26 \\
\hline $7,6,5,4$ & $3,2,1$ & 124 \\
\hline $7,6,5,4,2$ & 3,1 & 177 \\
\hline $7,6,5,4,2,3$ & 1 & 123 \\
\hline $7,6,5,4,2,3,1$ & - & 150 \\
\hline
\end{tabular}

Total: 628

Fuente: Elaboración propia

Comparando la ruta asignada por la empresa y la ruta propuesta por el método de expansión mínima, se obtiene el siguiente resultado: 


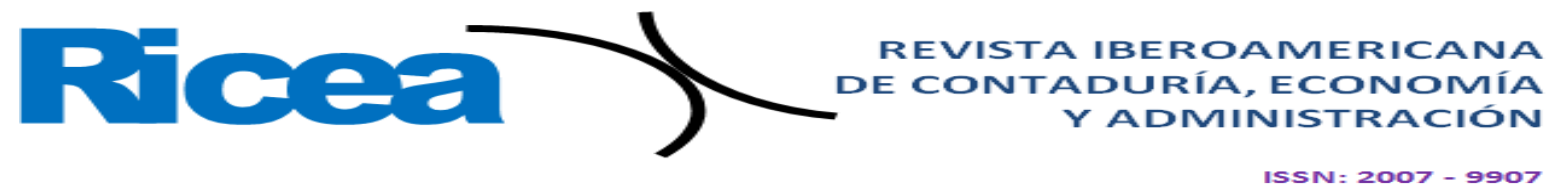

$$
\text { Resultado } 642 \mathrm{~km}-628 \mathrm{~km}=14 \mathrm{~km}
$$

Como se puede apreciar, con el método del árbol de expansión mínima se lograron disminuir $14 \mathrm{~km}$ en la ruta Huamantla-Tamanzunchale, trayecto en el cual deben entregar los pedidos solicitados por los clientes.

Igualmente, y para estimar el consumo de combustible en esta ruta, se tomó en cuenta el rendimiento del tráiler de 2.7 y el costo del diésel de \$12.70. En tal sentido, según el cálculo del consumo de combustible, y empleando el método de expansión mínima, a continuación se presenta la cantidad del costo de combustible que se redujo en la ida y el regreso de la ruta HuamantlaTamanzunchale:

$$
3019.80-2954.02=65.78(2)=\$ 131.56
$$

Consumo de combustible de la ruta asignada por la empresa:

consumo de combustible en litros $=\frac{642}{2.7}=237.78$ litros

costos de consumo de combustible $=\frac{642}{2.7}(12.70)=3019.80$

Consumo de combustible de la ruta obtenida por el método de árbol de expansión mínima:

consumo de combustible en litros $=\frac{628}{2.7}=232.60$ litros

costos de consumo de combustible $=\frac{628}{2.7}(12.70)=2954.02$ 


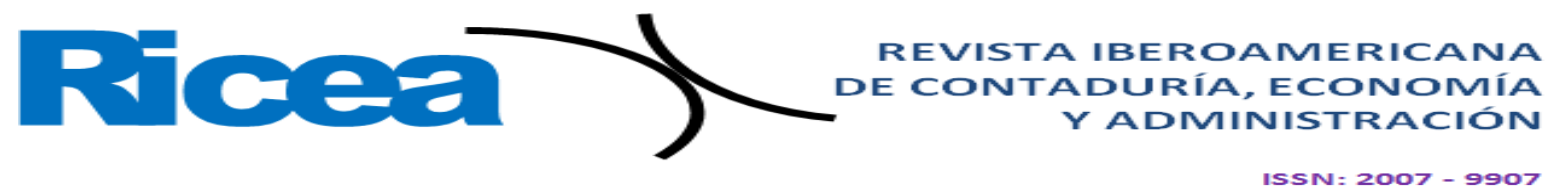

\section{Aplicación del modelo de transporte por el método de Vogel}

El presente modelo se basó en la demanda y oferta durante una semana del producto chechi kikis intermedio (queso-chile). En concreto, la oferta fue de 6480 bultos y la demanda de 5083 bultos por semana; los datos pueden variar según la cantidad de producto que el cliente requirió y los costos de traslado hacia sus destinos. La figura 11 muestra los datos recabados y calculados sobre la oferta y demanda del mencionado producto correspondiente a una semana, así como el costo que se genera al enviar los pedidos a sus respectivos destinos.

Figura 11. Datos recolectados de una semana para la aplicación del método Vogel

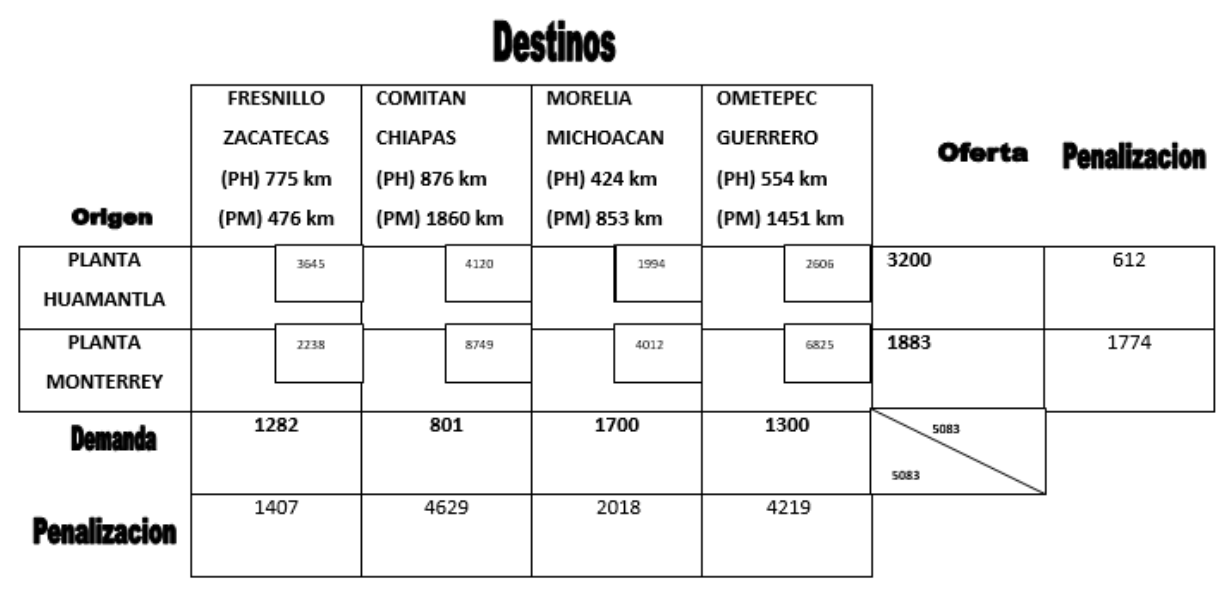

Fuente: Comercializadora Gonac (2012)

Por otra parte, y debido a que la capacidad de producción de ambas plantas es insuficiente para satisfacer la demanda, se tuvo que equilibrar la oferta y la demanda en la figura 11 con el fin de aplicar el método de Vogel.

En la figura 12 se presenta la solución del método de Vogel mediante el uso del programa Win QSB para poder asignar la cantidad de productos demandados a sus respectivos destinos al más bajo costo. Los costos suelen variar según los destinos y tipos de unidad que se envíen para entregar a los clientes, por lo que en este modelo se consideró el rendimiento de un tráiler de 2.7 y un costo aproximado de diésel de $\$ 12.70$ para todos los destinos. 


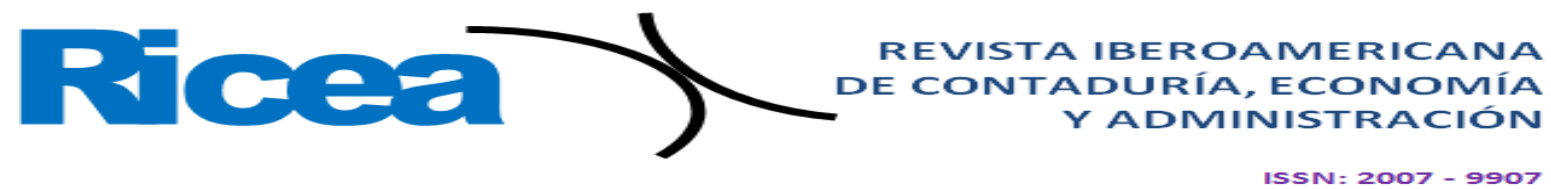

Figura 12. Solución del método Vogel aplicando el programa Win QSB

\begin{tabular}{|c|c|c|c|c|c|c|}
\hline \multicolumn{7}{|c|}{ 31. Transportation Tableau for Chechi Kikis Intermedio (queso-chile) - Iteration 1} \\
\hline From $\backslash \mathrm{T}_{0}$ & Destination & Destination & estination & estination 4 & Supply & Dual $\mathrm{P}(\mathrm{i})$ \\
\hline \multirow{2}{*}{ Source 1} & 3645 & 4120 & 1994 & 2606 & \multirow{2}{*}{3200} & \multirow{2}{*}{0} \\
\hline & $200^{*}$ & $\mathrm{Cij}=-6036 \times$ & 1700 & 1300 & & \\
\hline \multirow{2}{*}{ Source 2} & 2238 & 8749 & 4012 & 6825 & \multirow{2}{*}{1883} & \multirow{2}{*}{-1407} \\
\hline & 1082 & 801 & & & & \\
\hline Demand & 1282 & 801 & 1700 & 1300 & & \\
\hline \multirow[t]{3}{*}{ Dual $P_{(j)}$} & 3645 & 10156 & 1994 & 2606 & & \\
\hline & \multicolumn{6}{|c|}{ Objective Value $=1.693606 \mathrm{E}+07(\mathrm{Minimiz}$} \\
\hline & ${ }^{* k}$ Enterin & g: Source 1 to & Destinat & 12 * Lea & ng: Sou & 1 to Desti \\
\hline
\end{tabular}

Fuente: Elaboración propia

$$
\begin{gathered}
200(3645)+1700(1994)+1300(2606)+1082(2238)+801(8749)=16936065 \\
729000+3389800+3387800+2421516+7007949=16936065
\end{gathered}
$$

En la figura anterior se muestra cómo ambas plantas deben de satisfacer la demanda del mencionado producto para que los costos sean mínimos, así como la cantidad de productos que cada una de las plantas debe producir para atender la demanda requerida, lo cual puede ayudar a tomar mejores decisiones.

Las siguientes ventajas y desventajas hacen referencia al conjunto de métodos mencionados y aplicados en el presente artículo:

\section{Desventajas}

- Solo se consideran algunas variables como la oferta, la demanda, el tiempo, la distancia, el costo, los destinos para la optimización y la programación de rutas.

- No es posible considerar en el modelo de factores externos o fuera del alcance de la empresa. 


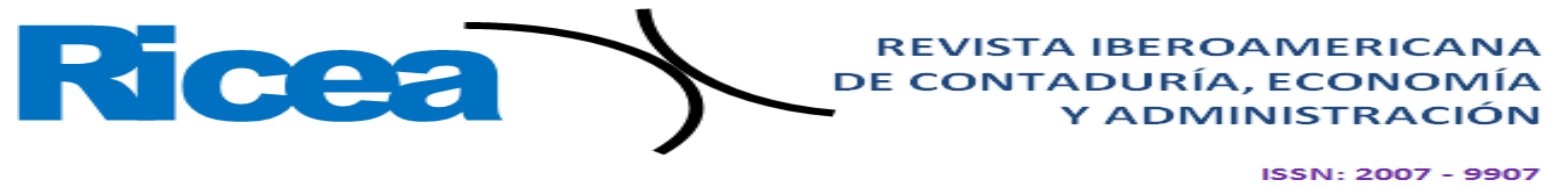

- No existen suficientes programas para la aplicación de estos modelos.

- Los cambios a los que se enfrentan las empresas.

\section{Ventajas}

- Flexibles para programar diferentes rutas.

- Fáciles de aplicar.

- Se puede complementar con mapas satelitales.

- Se utiliza en conjunto con un sistema de rastreo satelital GPS para cada unidad.

- Los resultados sirven como indicadores.

- Los modelos sirven como un sistema de control de transporte.

- Ayuda a tomar mejores decisiones estratégicamente.

- Hace que se satisfaga la demanda a tiempo.

- Permite la organización y coordinación de cada unidad con sus rutas o destinos.

- Reduce tiempos, costos y distancias de transporte.

- Los modelos se pueden controlar y programar desde un celular o tablet.

\section{Conclusiones}

La aplicación de las técnicas de optimización de redes y modelos de transporte permitió visualizar las ventajas y desventajas que se tienen al programar las diferentes rutas. En tal sentido, se debe prever que cada una de estas es diferente, por lo que las condiciones para programarlas y asignarlas varían, ya que se deben tomar en cuenta la cantidad y el volumen del producto solicitado por el cliente, así como el tiempo y la distancia recorrida, pues de esa manera se podrá escoger la unidad que debería trasladar determinado pedido a su destino, con lo cual se podrían disminuir los costos.

Por otro lado, vale comentar que los cambios a los que se encuentran expuestas las empresas las obligan a buscar oportunidades para reducir los costos de operatividad, específicamente en lo concerniente al transporte. 


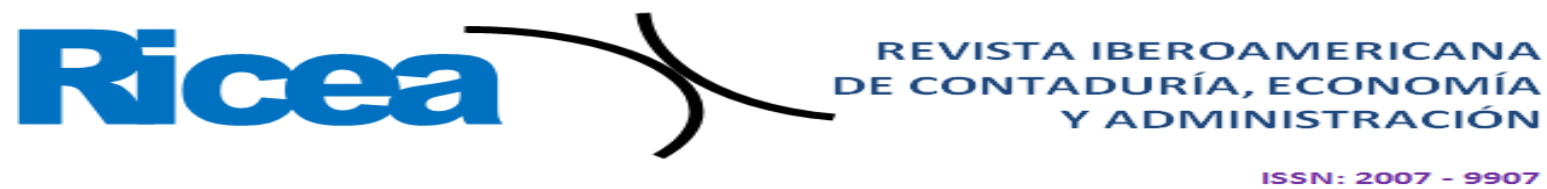

También se debe aceptar que los modelos usados en este trabajo pueden ser mejorados y adaptados, para lo cual se deben prever otras variables que interfieran en el proceso de optimización del transporte. De hecho, aún se debe desarrollar tecnología para camiones de carga pesada, pues en la actualidad se está trabajando con autos más pequeños.

Téngase en cuenta que el transporte de los insumos es un eslabón esencial en la cadena de operatividad de las empresas, lo cual dinamiza la economía de cualquier lugar. Además, este factor influye de manera notable en la fijación de precios de cualquier bien o servicio.

\section{Referencias}

Ballou, R. H. (2004). Logística: administración de la cadena de suministro. México: Pearson Educación.

Boluda Aguilar, M. y López Gómez, A. (2013). Producción de bioetanol mediante fermentación de subproductos cítricos pretratados con steam explosion (tesis doctoral). Cartagena (Colombia).

Castellanos Ramírez, A. (2009). Manual de gestión logística y distribución de mercancías. Barranquilla (Colombia): Unisorte.

Gay y García, C. (2014). Biocombustibles en México: una alternativa para la reducción de la dependencia de los hidrocarburos y la mitigación de los gases efecto invernadero. México: Universidad Nacional Autónoma de México.

Hiellier, F. L. (2006). Introducción a la investigación de operaciones. México: McGraw Hill.

Prawda, J. (2004). Métodos y modelos de investigación de operaciones I. México: Limusa.

Taha, H. A. (2004). Investigación de operaciones. México: Pearson Educación. 


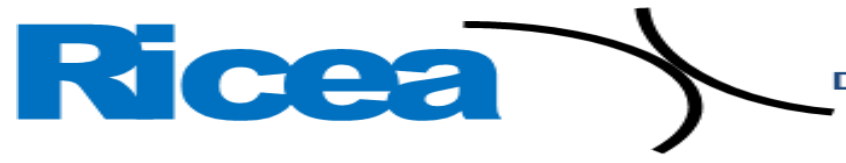

REVISTA IBEROAMERICANA DE CONTADURÍA, ECONOMÍA YADMINISTRACIÓN

ISSN = $2007-9907$

\begin{tabular}{|c|c|}
\hline Rol de Contribución & Autor (es) \\
\hline Conceptualización & $\begin{array}{l}\text { María Elizabeth Serrano Cervantes y Luis de Jesús Montero Garcia (grado de } \\
\text { contribución igual) }\end{array}$ \\
\hline Metodología & $\begin{array}{l}\text { María Elizabeth Serrano Cervantes y Luis de Jesús Montero Garcia (grado de } \\
\text { contribución igual) }\end{array}$ \\
\hline Software & $\begin{array}{l}\text { María Elizabeth Serrano Cervantes y Luis de Jesús Montero Garcia (grado de } \\
\text { contribución igual) }\end{array}$ \\
\hline Validación & $\begin{array}{l}\text { María Elizabeth Serrano Cervantes y Luis de Jesús Montero Garcia (grado de } \\
\text { contribución igual) }\end{array}$ \\
\hline Análisis Formal & $\begin{array}{l}\text { María Elizabeth Serrano Cervantes y Luis de Jesús Montero Garcia (grado de } \\
\text { contribución igual) }\end{array}$ \\
\hline Investigación & $\begin{array}{l}\text { María Elizabeth Serrano Cervantes y Luis de Jesús Montero Garcia (grado de } \\
\text { contribución igual) }\end{array}$ \\
\hline Recursos & No aplica \\
\hline Curación de datos & $\begin{array}{l}\text { María Elizabeth Serrano Cervantes y Luis de Jesús Montero Garcia (grado de } \\
\text { contribución igual) }\end{array}$ \\
\hline $\begin{array}{l}\text { Escritura - Preparación del } \\
\text { borrador original }\end{array}$ & $\begin{array}{l}\text { María Elizabeth Serrano Cervantes y Luis de Jesús Montero Garcia (grado de } \\
\text { contribución igual) }\end{array}$ \\
\hline Escritura - Revisión y edición & $\begin{array}{l}\text { María Elizabeth Serrano Cervantes y Luis de Jesús Montero Garcia (grado de } \\
\text { contribución igual) }\end{array}$ \\
\hline Visualización & $\begin{array}{l}\text { María Elizabeth Serrano Cervantes y Luis de Jesús Montero Garcia (grado de } \\
\text { contribución igual) }\end{array}$ \\
\hline Supervisión & $\begin{array}{l}\text { María Elizabeth Serrano Cervantes y Luis de Jesús Montero Garcia (grado de } \\
\text { contribución igual) }\end{array}$ \\
\hline Administración de Proyectos & $\begin{array}{l}\text { María Elizabeth Serrano Cervantes y Luis de Jesús Montero Garcia (grado de } \\
\text { contribución igual) }\end{array}$ \\
\hline Adquisición de fondos & $\begin{array}{l}\text { María Elizabeth Serrano Cervantes y Luis de Jesús Montero Garcia (grado de } \\
\text { contribución igual) }\end{array}$ \\
\hline
\end{tabular}

